\title{
Impact of Apolipoprotein B on Hepatosteatosis in a Population Infected with Hepatitis C Virus: A Cross-Sectional Observational Study
}

\author{
Ming-Shyan Lin ${ }^{a}$ Su-Er Guob,c, d Huang-Shen Lin ${ }^{\mathrm{e}}$ Jen-Te Hsu ${ }^{f}$ \\ Yu-Sheng Lin ${ }^{f}$ Tsai-Hui Ling Tung-Jung Huang ${ }^{b}$ h Mei-Yen Chen ${ }^{b}$ \\ Chang-Min Chung ${ }^{b}$ f \\ a Division of Cardiology, Chang Gung Memorial Hospital, Yunlin, Taiwan; ${ }^{b}$ College of Nursing \\ \& Graduate Institute of Nursing, Chang Gung University of Science and Technology (CGUST), \\ Yunlin, Taiwan; ${ }^{\circ}$ Chronic Diseases \& Health Promotion Research Center, Chang Gung University \\ of Science and Technology (CGUST), Yunlin, Taiwan; ${ }^{\mathrm{d}}$ Research Center for Industry of Human \\ Ecology, Chang Gung University of Science and Technology (CGUST), Yunlin, Taiwan; ${ }^{\text {e Division }}$

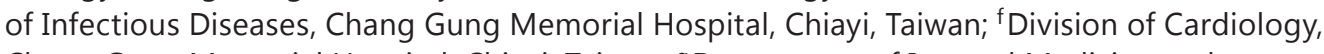 \\ Chang Gung Memorial Hospital, Chiayi, Taiwan; ${ }^{9}$ Department of Internal Medicine and \\ Traditional Chinese Medicine, China Medical University Hospital, Taichung, Taiwan; h Division of \\ Pulmonary Disease, Chang Gung Memorial Hospital, Yunlin, Taiwan
}

\section{Key Words}

Hepatitis C virus · Apolipoprotein-B · Non-alcoholic fatty liver disease

\begin{abstract}
Objective: Non-alcoholic fatty liver disease (NAFLD) is an established risk factor for diabetes, cardiovascular disease, antiviral treatment resistance, and progression of chronic hepatitis $C$ virus (HCV) infection to fibrosis. Apolipoprotein-B 100 (ApoB-100) is a dyslipidemia marker and steatosis predictor. We assess the correlation between ApoB-100 and hepatosteatosis. Methods: This cross-sectional study enrolled 1,218 HCV-seropositive participants from a 2012-2013 health checkup in Taiwan. NAFLD was detected using ultrasound. All anthropometric and laboratory studies that included ApoB-100 were evaluated whether or not ApoB100 predicts NAFLD. Logistic regression was also used to examine the association between ApoB-100 and NAFLD. Results: Participants were $47.16 \pm 16.08$ years old (mean age). The overall prevalence of NAFLD was $35.8 \%$ ( $n=436 ; 32.8 \%$ men, $38.1 \%$ women). Participants with ApoB-100 $\geq 8$ had a significantly higher incidence of NAFLD (39.4 vs. $29.4 \%$; $95 \%$ CI $0.044-$ $0.156 ; p<0.001)$. After confounding factors had been adjusted for, ApoB-100 was significant-
\end{abstract}

Chang-Min Chung and Mei-Yen Chen contributed equally to this paper.

Dr. Chang-Min Chung

Division of Cardiology

Chang Gung Memorial Hospital

6, Sec. West Chai-Pu Road, Pu-TZ City, 61363 Chiayi, Taiwan

myheart88168@gmail.com 
Lin et al.: Impact of Apolipoprotein B on Hepatosteatosis in a Population Infected with Hepatitis C Virus: A Cross-Sectional Observational Study

ly associated with NAFLD (OR 5.45; 95\% CI 1.64-18.06; $p=0.006)$ and high-grade hepatosteatosis (OR 7.73; 95\% CI 1.74-34.35; $p=0.007$ ). Conclusion: ApoB-100 is strongly associated with NAFLD in people with non-genotype $3 \mathrm{HCV}$; greater ApoB-100 content is significantly correlated with higher-grade hepatosteatosis.

(C) 2016 The Author(s)

Published by S. Karger GmbH, Freiburg

\section{Introduction}

Hepatitis C virus (HCV) infection is an emerging health concern with an estimated global prevalence of $3 \%$ and 185 million chronic carriers worldwide [1]. Chronic HCV progresses from steatosis over fibrosis to cirrhosis and hepatocellular carcinoma. In addition, HCV increases insulin resistance and the incidence of metabolic syndrome and diabetes mellitus (DM) $[2,3]$, all of which contribute to the extrahepatic complications of systemic atherosclerosis and coronary artery disease [4].

Hepatosteatosis in chronic hepatitis C (CHC) is estimated to have a prevalence of 55-81\%, which is significantly higher than its overall prevalence $(15-40 \%)[5,6]$. Non-alcoholic fatty liver disease (NAFLD) is significantly associated with metabolic syndrome [7], which increases necroinflammation [8], accelerate the development of fibrosis, increases refractoriness to anti-viral therapy [9], and causes early atherosclerosis [10]. Therefore, the complex relationships between hepatosteatosis, insulin resistance, hepatic fibrosis and systemic inflammation contribute to higher mortality in CHC [11].

Many atherolipid profiles are associated with NAFLD. Apolipoprotein-B100 (ApoB-100) is the primary protein responsible for carrying cholesterol, including very low-density lipoprotein (VLDL) and low-density lipoprotein (LDL), to tissue, which might be inaccurately measured in hypertriglyceridemia and NAFLD $[12,13]$. Studies have reported a strong association between ApoB, metabolic syndrome, DM [14] and cardiovascular events [15-17]. In addition, ApoB-100 is also a predictor of NAFLD in patients with [18] and without DM [19]. Despite the hypobetalipoproteinemia with hypocholesteremia observed in patients with genotype-3 HCV [20], there was no additional evidence of a correlation between ApoB-100 and steatosis in non-genotype 3 CHC.

To examine the involvement of ApoB-100 in HCV-related hepatosteatosis, we investigated the association between various measures of body weight (BW), BMI, waist-to-hip ratio (WHR), metabolic syndrome, and NAFLD in non-genotype 3 HCV patients.

\section{Material and Methods}

\section{Sample and Study Design}

The present study is a cross-sectional study based on an annual community health checkup. We aimed to determine the relationship between associated factors and hepatosteatosis in an HCV hyperendemic village. From the end of 2012 until August 2013, the health checkup screened 12,348 residents. The eligibility criteria for the present study included being > 20 years old and HCV-seropositive. Exclusion criteria were an incomplete health checklist as well as missing anthropometric records, laboratory data, and hepatitis viral markers, or a missing echogenic study. Finally, 1,218 eligible residents were identified and enrolled in the analysis (fig. 1). Ethical approval was granted by the hospital's Institutional Review Board (IRB: 103-6854B) 
Fig. 1. Flow chart of inclusion cri-

Lin et al.: Impact of Apolipoprotein B on Hepatosteatosis in a Population Infected with Hepatitis C Virus: A Cross-Sectional Observational Study

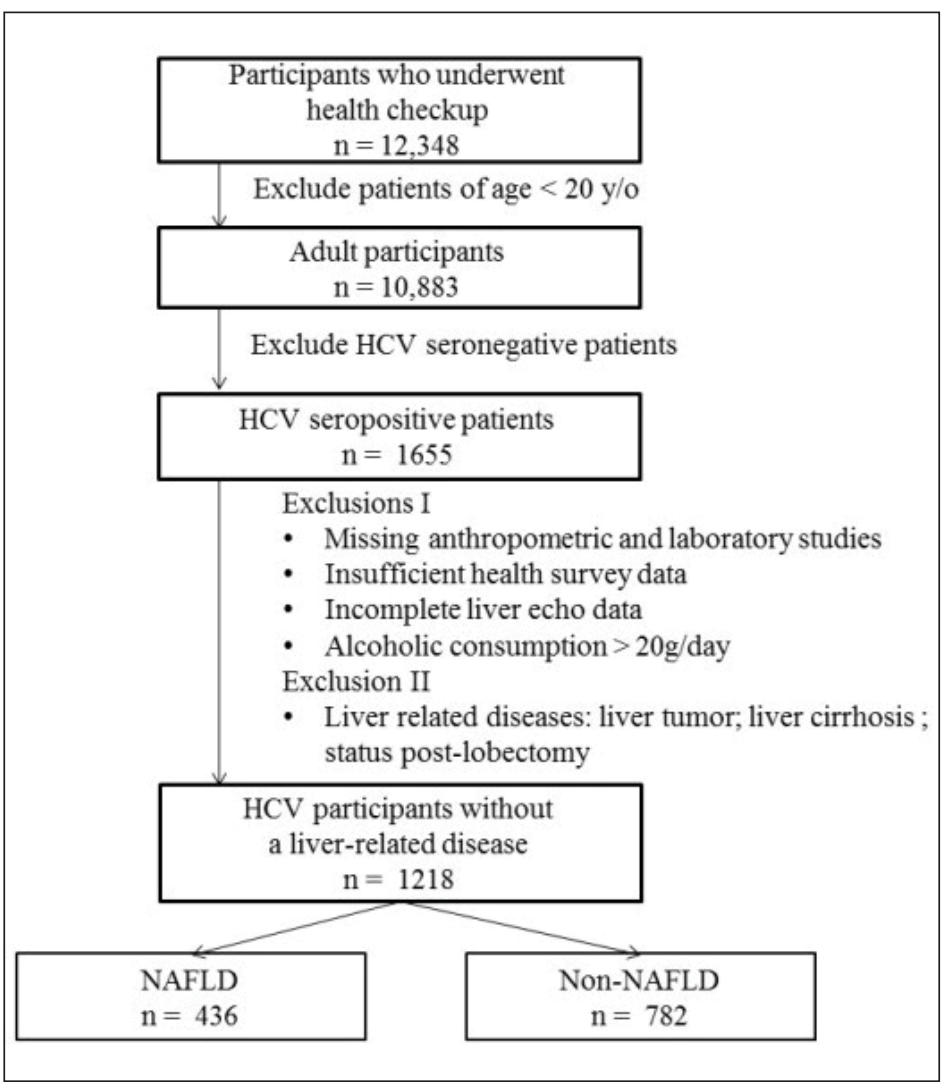

\section{Anthropometric, Blood Pressure, and Laboratory Measurements}

Three measurements of waist circumference were taken midway between the lowest rib and the iliac crest to an accuracy of $0.5 \mathrm{~cm}$. The hip was measured at the widest portion on the left side, and at the greater trochanters on the right. Both were calculated as the WHR. Measurements of weight and height were also taken to calculate the BMI: weight $(\mathrm{kg}) /$ height $\left(\mathrm{m}^{2}\right)$. Blood pressure was measured using a standard electronic sphygmomanometer according to the Hypertension Detection and Follow-up Program protocol (HDFP) while the patient was sitting comfortably with their back supported for at least 5 min. Pulse pressure was calculated as systolic blood pressure (SBP) - diastolic blood pressure (DBP).

After the participants had fasted for at least $12 \mathrm{~h}$, their blood was drawn for biochemical analyses of serum glucose, creatinine, uric acid, triglycerides, total cholesterol, high-density lipoprotein (HDLLDL, VLDL, alkaline phosphatase, alanine aminotransferase (ALT), aspartate aminotransferase, gamma-glutamyl transferase, and ApoB-100 using an 7600 automatic biochemistry analyzer (Model 7699; Hitachi, Tokyo, Japan). ApoB-100 concentrations were measured with the Tina-quant apoB kits (Roche Diagnostics, Taipei, Taiwan) (version with the International Federation of Clinical Chemistry SP3-07 reference preparations) according to World Health Organization standardized methods [21].

Hepatitis B virus (HBV) and HCV seropositivity was assessed using sandwich radioimmunoassay (Elecsys; Cobas analyzer; Roche Diagnostics), a semiquantitative method to determine hepatitis B surface antigen and antibody to HCV using an electrochemiluminescence immunoassay test (Roche Diagnostics), respectively.

\section{Personal Medical History}

Participants completed a health checklist for baseline disease and lifestyle habits: smoking, alcohol drinking, and betel nut chewing. DM was defined by a participant's having a clinical diagnosis of the disease or by documented use of anti-diabetes drugs. Hypertension was defined by a participant's having a clinical diagnosis of high blood pressure or by documented use of anti-hypertension medication. Significant 
Lin et al.: Impact of Apolipoprotein B on Hepatosteatosis in a Population Infected with Hepatitis C Virus: A Cross-Sectional Observational Study

alcohol consumption was defined as ongoing or recent alcohol consumption $>20 \mathrm{~g} /$ day. A smoker was defined as someone who had smoked at least 100 cigarettes in their lifetime and was a current smoker at the time of the study. Finally, a betel nut chewer was defined as someone who reported chewed betel nut $>$ once a week

\section{Abdominal Echography}

The ultrasound examinations of supine patients were done using three units with curved array transducers: Aloka SSD 4000 + UST-979-3.5 transducer (Hitachi Aloka Medical, Wallingford, CT, USA), Acuson S2000 + C4-1 MHz transducer (Siemens Limited, Taipei, Taiwan) and a CGM OPUS 5000 + CLA35 transducer (Chang Gung Medical Technology, Taipei, Taiwan). Technical parameters were adjusted for each patient based on a standard protocol. Livers with a homogenous echo texture, no acoustic attenuation, visible portal veins, a well-visualized diaphragm, and an echogenicity similar to or slightly higher than that of renal parenchyma were considered normal [22]. The diagnosis of the fatty liver was based on the brightness of the liver in comparison to the kidney, vascular blurring of hepatic vein trunk, and deep attenuation in the right hepatic lobe. The severity of fatty liver change was divided into four grades (0-3): Grade $0=$ normal liver, normal echo texture, and no fatty change; Grade 1 = mild fatty liver change, mild increase in fine echoes in the parenchyma with slightly impaired visualization of the intrahepatic vessels and diaphragm; Grade $2=$ medium grade diffuse increase in hepatic echogenity, mild deterioration in the image of the diaphragm and intrahepatic vessels; Grade 3 = moderate to severe fatty liver change, marked increase in fine echoes in the parenchyma with poor or non-visualization of the intrahepatic vessel borders, diaphragm and posterior right lobe of the liver. All images were reviewed on a picture archiving and communication system (Centricity PACS; GE Healthcare, Ridgewood, NJ, USA).

\section{Definitions}

\section{NAFLD}

The definition of NAFLD requires i) evidence of hepatic steatosis by imaging and ii) the absence of secondary causes for hepatic fat accumulation such as significant alcohol consumption, use of steatogenic medication, or hereditary disorders. Low-grade NAFLD means grade 1 hepatosteatosis, and high-grade NAFLD means grades 2-3 hepatosteatosis.

\section{Metabolic Syndrome}

Metabolic syndrome based on the 2006 National Cholesterol Education Program Adult Treatment Panel III guideline [23] was defined as evidence of three or more of the following: i) waist circumference $>90 \mathrm{~cm}$ in men and $>80 \mathrm{~cm}$ in women for Asians; ii) fasting plasma glucose $\geq 110 \mathrm{mg} / \mathrm{dl}$ or taking anti-diabetes medication; iii) serum triglycerides $\geq 150 \mathrm{mg} / \mathrm{dl}$; iv) serum $\mathrm{HDL}$-cholesterol $<40 \mathrm{mg} / \mathrm{dl}$ in men and $<50 \mathrm{mg} / \mathrm{dl}$ in women; v) blood pressure $\geq 130 / 85$ mm Hg or taking anti-hypertension medication.

\section{Statistical Analyses}

SPSS 19.0 for Windows (SPSS, Inc., Chicago, IL, USA) was used for all analyses. Baseline characteristics and laboratory findings were compared between HCV-seropositive participants based on NAFLD and ApoB-100 level. Continuous variables, reported as means \pm standard deviation (SD), were compared using Student's t test. Categorical variables are reported as number (\%) and were compared using Fisher's Exact test. Factors associated with NAFLD were identified using a multivariate logistic regression analysis. Significance was set at $\mathrm{p}<0.05$ (two-sided). 
Lin et al.: Impact of Apolipoprotein B on Hepatosteatosis in a Population Infected with Hepatitis C Virus: A Cross-Sectional Observational Study

Table 1. Baseline characteristics differences between HCV seropositive patients with or without NAFL

\begin{tabular}{|c|c|c|c|c|}
\hline \multirow[t]{2}{*}{ Characteristic } & \multirow[t]{2}{*}{ All (n = 1,218) } & \multicolumn{2}{|l|}{ NAFLD } & \multirow[t]{2}{*}{$\mathrm{p}$ values } \\
\hline & & yes $(n=436)$ & no $(\mathrm{n}=782)$ & \\
\hline Age,years & $47.16 \pm 16.08$ & $47.62 \pm 15.89$ & $46.90 \pm 16.19$ & 0.459 \\
\hline Gender, female, n (\%) & $696(57.1 \%)$ & $265(60.8 \%)$ & $431(55.1 \%)$ & 0.055 \\
\hline BW, kg & $65.89 \pm 13.23$ & $66.19 \pm 13.27$ & $65.73 \pm 13.22$ & 0.562 \\
\hline $\mathrm{BMI} \pm \mathrm{SD}$ & $25.02 \pm 4.12$ & $25.24 \pm 4.15$ & $24.90 \pm 4.09$ & 0.163 \\
\hline $\mathrm{BMI}>25, \mathrm{n}(\%)$ & $577(47.4 \%)$ & $214(49.1 \%)$ & $363(46.4 \%)$ & 0.372 \\
\hline Waist circumference, $\mathrm{cm}$ & $82.54 \pm 11.23$ & $82.86 \pm 11.06$ & $82.36 \pm 11.32$ & 0.455 \\
\hline $\mathrm{WHR} \pm \mathrm{SD}$ & $0.86 \pm 0.08$ & $0.86 \pm 0.09$ & $0.86 \pm 0.08$ & 0.908 \\
\hline SBP, mm Hg & $135.90 \pm 20.65$ & $138.12 \pm 19.91$ & $134.66 \pm 20.97$ & 0.005 \\
\hline DBP, mm Hg & $80.72 \pm 14.27$ & $82.30 \pm 13.52$ & $79.84 \pm 14.60$ & 0.004 \\
\hline Pulse pressure, mm Hg & $55.17 \pm 13.94$ & $55.82 \pm 13.56$ & $54.82 \pm 14.14$ & 0.230 \\
\hline Diabetes mellitus, n (\%) & $144(11.8 \%)$ & $88(20.2 \%)$ & $56(7.2 \%)$ & $<0.001$ \\
\hline Hypertension, n (\%) & $249(20.4 \%)$ & $132(30.3 \%)$ & $117(15.0 \%)$ & $<0.001$ \\
\hline HBV, n (\%) & $180(14.8 \%)$ & $61(14.0 \%)$ & $119(15.2 \%)$ & 0.563 \\
\hline Smoking, n (\%) & $131(10.8 \%)$ & $59(13.5 \%)$ & $72(9.5 \%)$ & 0.020 \\
\hline Betel-nut chewing, n (\%) & $75(6.2 \%)$ & $36(8.3 \%)$ & $39(5.0 \%)$ & 0.023 \\
\hline Creatinine, $\mathrm{mg} / \mathrm{dl}$ & $0.93 \pm 0.33$ & $0.92 \pm 0.40$ & $0.93 \pm 0.28$ & 0.673 \\
\hline Fasting glucose, $\mathrm{mg} / \mathrm{dl}$ & $109.93 \pm 34.01$ & $114.44 \pm 36.84$ & $107.41 \pm 32.07$ & 0.001 \\
\hline ALT, mg/dl & $40.30 \pm 49.89$ & $41.14 \pm 64.12$ & $39.83 \pm 39.86$ & 0.662 \\
\hline AST, mg/dl & $34.57 \pm 32.31$ & $33.42 \pm 36.33$ & $35.21 \pm 29.84$ & 0.355 \\
\hline Uric acid, mg/dl & $5.99 \pm 1.60$ & $6.08 \pm 1.61$ & $5.94 \pm 1.59$ & 0.126 \\
\hline LDL-C, mg/dl & $113.63 \pm 31.13$ & $115.66 \pm 31.28$ & $112.49 \pm 31.02$ & 0.089 \\
\hline HDL-C, mg/dl & $51.01 \pm 13.10$ & $49.04 \pm 12.18$ & $52.10 \pm 13.47$ & $<0.001$ \\
\hline $\mathrm{TC}, \mathrm{mg} / \mathrm{dl}$ & $184.20 \pm 36.47$ & $187.09 \pm 37.90$ & $182.58 \pm 35.57$ & 0.038 \\
\hline $\mathrm{TG}, \mathrm{mg} / \mathrm{dl}$ & $111.35 \pm 67.56$ & $129.66 \pm 87.87$ & $101.15 \pm 50.21$ & $<0.001$ \\
\hline GGT, U/l & $35.46 \pm 55.99$ & $36.59 \pm 43.46$ & $34.83 \pm 61.91$ & 0.600 \\
\hline ApoB-100, g/l & $0.88 \pm 0.23$ & $0.91 \pm 0.24$ & $0.86 \pm 0.22$ & $<0.001$ \\
\hline MetS, n (\%) & $346(28.4 \%)$ & $163(37.4 \%)$ & $183(23.4 \%)$ & $<0.001$ \\
\hline
\end{tabular}

Values are number (\%) or mean \pm SD; Fisher's Exact test for categorical variables and one-way ANOVA for continuous variables.

ApoB-100 = apolipoprotein-B 100; ALT = alanine aminotransferase; AST = aspartate aminotransferase; GGT = gamma-glutamyl transferase; LDL-C = low-density lipoprotein-cholesterol; HDL-C = high-density lipoprotein-cholesterol; $\mathrm{TC}=$ total cholesterol; $\mathrm{TG}=$ triglyceride .

\section{Results}

\section{Study Population}

After we had excluded patients who had not met the inclusion criteria, 1,218 enrollees were included for the final analysis (mean age $47.16 \pm 16.08$ years; females $57.1 \%$ ). The prevalence of DM, hypertension, metabolic syndrome and BMI $>25 \mathrm{~kg} / \mathrm{m}^{2}$ was $11.8 \%(\mathrm{n}=144)$, $20.4 \%(n=249), 28.4 \%(n=346)$ and $47.4 \%(n=577)$, respectively. The participants were divided into the NAFLD $(n=436)$ and non-NAFLD $(n=782)$ groups (table 1$)$. There were nonsignificantly more women in the NAFLD group (60.8\% vs. $55.1 \%$; $=0.055)$. Despite significantly higher percentages of DM, hypertension $(p<0.001)$, smoking $(p=0.020)$, betel-nut chewing $(p=0.023)$ in the NAFLD group, age, WHR, BW, and BMI were not significantly different. Patients with NAFLD had typical metabovascular features with significantly lower HDL (mean $49.04 \pm 12.18$ vs. $52.10 \pm 13.47 \mathrm{mg} / \mathrm{dl} ; \mathrm{p}<0.001$ ) but significantly higher total cholesterol (187.09 \pm 37.90 vs. $182.58 \pm 35.57 \mathrm{mg} / \mathrm{dl} ; \mathrm{p}=0.038$ ), triglycerides (129.66 \pm 87.87 vs. $101.15 \pm 50.21 \mathrm{mg} / \mathrm{dl} ; \mathrm{p}<0.001$ ), fasting glucose (mean $114.44 \pm 36.84$ vs. 
Lin et al.: Impact of Apolipoprotein B on Hepatosteatosis in a Population Infected with Hepatitis C Virus: A Cross-Sectional Observational Study

Table 2. Baseline characteristics differences between patients with HCV based on ApoB-100 content

\begin{tabular}{|c|c|c|c|}
\hline Characteristic & $\begin{array}{l}\text { АроВ } \geq 0.8 \\
(n=782)\end{array}$ & $\begin{array}{l}\text { АроB }<0.8 \\
(n=436)\end{array}$ & $\mathrm{p}$ values \\
\hline Age, years & $47.64 \pm 16.01$ & $46.30 \pm 16.20$ & 0.164 \\
\hline Gender, female, n (\%) & $440(56.3 \%)$ & $256(58.7 \%)$ & 0.408 \\
\hline BW, kg & $66.19 \pm 13.14$ & $65.36 \pm 13.40$ & 0.299 \\
\hline BMI & $25.11 \pm 4.07$ & $24.85 \pm 4.19$ & 0.298 \\
\hline $\mathrm{BMI}>25, \mathrm{n}(\%)$ & $364(46.5 \%)$ & $213(48.9)$ & 0.440 \\
\hline Waist circumference, $\mathrm{cm}$ & $82.86 \pm 11.25$ & $81.97 \pm 11.18$ & 0.184 \\
\hline WHR & $0.86 \pm 0.08$ & $0.85 \pm 0.08$ & 0.104 \\
\hline $\mathrm{SBP}, \mathrm{mm} \mathrm{Hg}$ & $136.67 \pm 20.74$ & $134.51 \pm 20.45$ & 0.080 \\
\hline $\mathrm{DBP}, \mathrm{mm} \mathrm{Hg}$ & $81.28 \pm 14.43$ & $79.72 \pm 13.93$ & 0.066 \\
\hline Pulse pressure, mm Hg & $55.39 \pm 14.17$ & $54.79 \pm 13.52$ & 0.477 \\
\hline Diabetes mellitus, n (\%) & $84(10.7 \%)$ & $60(13.8 \%)$ & 0.118 \\
\hline Hypertension, $\mathrm{n}(\%)$ & $172(22.0 \%)$ & $77(17.7 \%)$ & 0.072 \\
\hline HBV, n (\%) & $121(15.5 \%)$ & $59(13.5 \%)$ & 0.360 \\
\hline Smoking, n (\%) & $79(10.1 \%)$ & $52(11.9 \%)$ & 0.325 \\
\hline Betel-nut chewing, n (\%) & $48(6.1 \%)$ & $27(6.2 \%)$ & 0.970 \\
\hline Creatinine, $\mathrm{mg} / \mathrm{dl}$ & $0.94 \pm 0.36$ & $0.91 \pm 0.27$ & 0.182 \\
\hline Fasting glucose, $\mathrm{mg} / \mathrm{dl}$ & $110.64 \pm 34.45$ & $108.64 \pm 33.19$ & 0.323 \\
\hline ALT, mg/dl & $44.60 \pm 44.31$ & $37.90 \pm 52.63$ & 0.025 \\
\hline AST, mg/dl & $39.64 \pm 35.49$ & $31.74 \pm 30.04$ & $<0.001$ \\
\hline Uric acid, mg/dl & $6.11 \pm 1.62$ & $5.78 \pm 1.55$ & 0.001 \\
\hline $\mathrm{LDL}, \mathrm{mg} / \mathrm{dl}$ & $129.01 \pm 26.20$ & $86.05 \pm 17.07$ & $<0.001$ \\
\hline HDL, mg/dl & $49.82 \pm 12.32$ & $53.13 \pm 14.16$ & $<0.001$ \\
\hline $\mathrm{TC}, \mathrm{mg} / \mathrm{dl}$ & $200.80 \pm 31.75$ & $154.42 \pm 22.97$ & $<0.001$ \\
\hline $\mathrm{TG}, \mathrm{mg} / \mathrm{dl}$ & $125.35 \pm 70.41$ & $86.24 \pm 53.72$ & $<0.001$ \\
\hline GGT, U/l & $34.13 \pm 47.92$ & $37.86 \pm 68.12$ & 0.265 \\
\hline MetS, n (\%) & $256(32.7 \%)$ & $90(20.6 \%)$ & $<0.001$ \\
\hline
\end{tabular}

Values are number $(\%)$ or mean \pm SD; Fisher's Exact test for categorical variables and one-way ANOVA for continuous variables.

ALT = Alanine aminotransferase; AST = aspartate aminotransferase; GGT = gamma-glutamyl transferase; LDL-C = low-density lipoprotein-cholesterol; HDL-C = high-density lipoprotein-cholesterol; $\mathrm{TC}=$ total cholesterol; $\mathrm{TG}=$ triglyceride.

$107.41 \pm 32.07 \mathrm{mg} / \mathrm{dl} ; \mathrm{p}=0.001)$, higher SBP $(138.12 \pm 19.91$ vs. $134.66 \pm 20.97 \mathrm{~mm} \mathrm{Hg} ; \mathrm{p}=$ $0.005)$, DBP ( $82.30 \pm 13.52$ vs. $79.84 \pm 14.60 \mathrm{~mm} \mathrm{Hg}$; $=0.004)$, and ApoB-100 content (0.91 \pm 0.24 vs. $0.86 \pm 0.22 \mathrm{~g} / \mathrm{l} ; \mathrm{p}<0.001$ ).

Nonsignificantly more women had ApoB-100 content $\geq$ and $<0.8(56.3 \%$ and $58.7 \%$; $p=$ 0.408) (table 2). Age distribution, BW, BMI, waist size, and WHR were not significantly different. The proportion of metabolic syndrome was significantly higher in patients with ApoB-100 $\geq 0.8$ (32.7 vs. 20.6\%; p < 0.001); these patients also had significantly higher serum LDL, total cholesterol, triglycerides, and significantly lower HDL. However, SBP, DBP, pulse pressure, serum creatinine, fasting glucose, and GGT were not significantly different.

The incidences of NAFLD (39.4 vs. 29.4\%; $\mathrm{p}<0.001$ ) and high-grade NAFLD (17.3 vs. $11.0 \%$; $=0.003$ ) were significantly higher in patients with ApoB-100 $\geq 0.8$ (fig. 2), and the mean value of ApoB-100 significantly increased at higher grades of NAFLD: Grade $1=0.86$ g/l, Grade $2=0.88$ g/l, Grade $3=0.95$ g/l; p $<0.001$ (fig. 3). 
Fig. 2. Incidence of NAFLD and high-grade hepatosteatosis based on the ApoB-100 level.

Lin et al.: Impact of Apolipoprotein B on Hepatosteatosis in a Population Infected with Hepatitis C Virus: A Cross-Sectional Observational Study

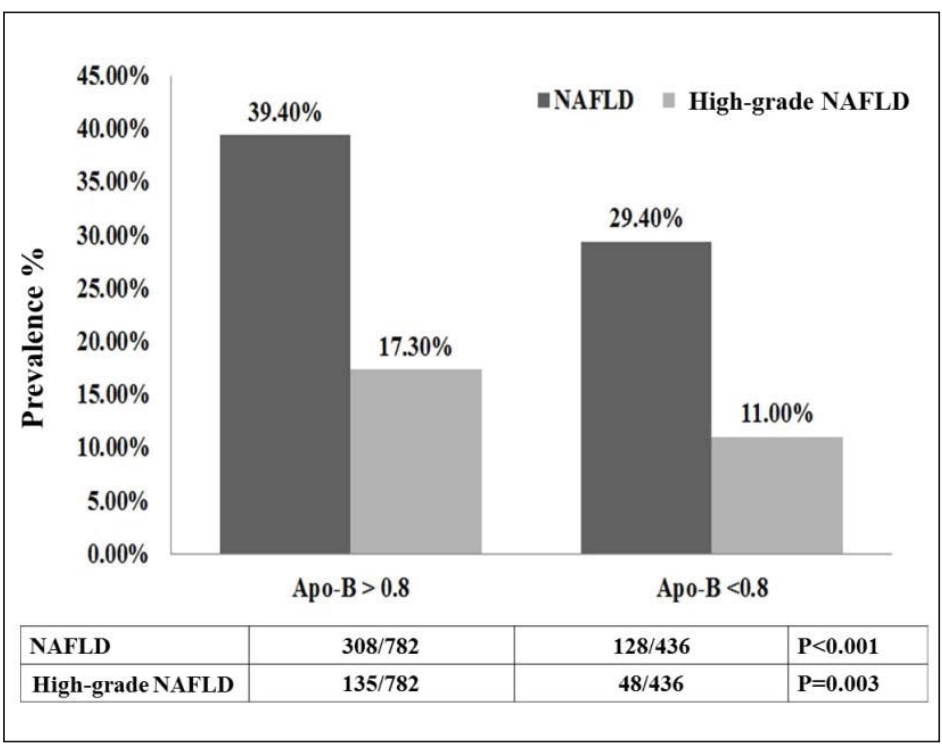

Fig. 3. The ApoB-100 levels by NAFLD grade.

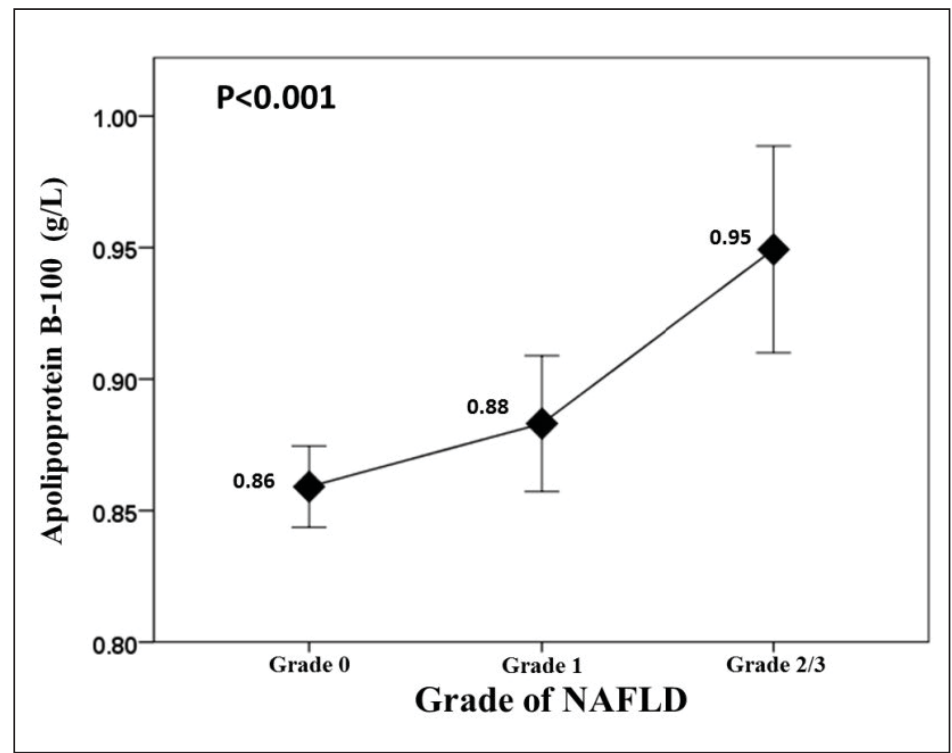

Independent Factors of Hepatosteatosis in HCV-Seropositive Patients

All variables were evaluated for predicting NAFLD and high-grade hepatosteatosis. ApoB-100 (OR 5.45; 95\% CI 1.64-18.06; $\mathrm{p}=0.006$;) and metabolic syndrome (OR 1.58; 95\% CI 1.18-2.11; $\mathrm{p}=0.002$ ) were independently associated with NAFLD (Grades 1-3) (fig. 4A), and ApoB-100 (OR 7.73; 95\% CI 1.64-34.35; p = 0.006), metabolic syndrome (OR 1.79; 95\% CI 1.24-2.59; $\mathrm{p}=0.002$ ), and uric acid (OR 1.17; 95\% CI 1.05-1.31; $\mathrm{p}=0.006$ ) were strongly correlated with high-grade NAFLD (grades 2-3) (fig. 4B).

\section{Discussion}

This is the first cross-sectional population study that shows a strong association between ApoB-100 and NAFLD in an HCV-seropositive population. The higher ApoB-100 levels indicated a significant risk (OR: 7.73) for high-grade hepatosteatosis. 
Lin et al.: Impact of Apolipoprotein B on Hepatosteatosis in a Population Infected with Hepatitis C Virus: A Cross-Sectional Observational Study

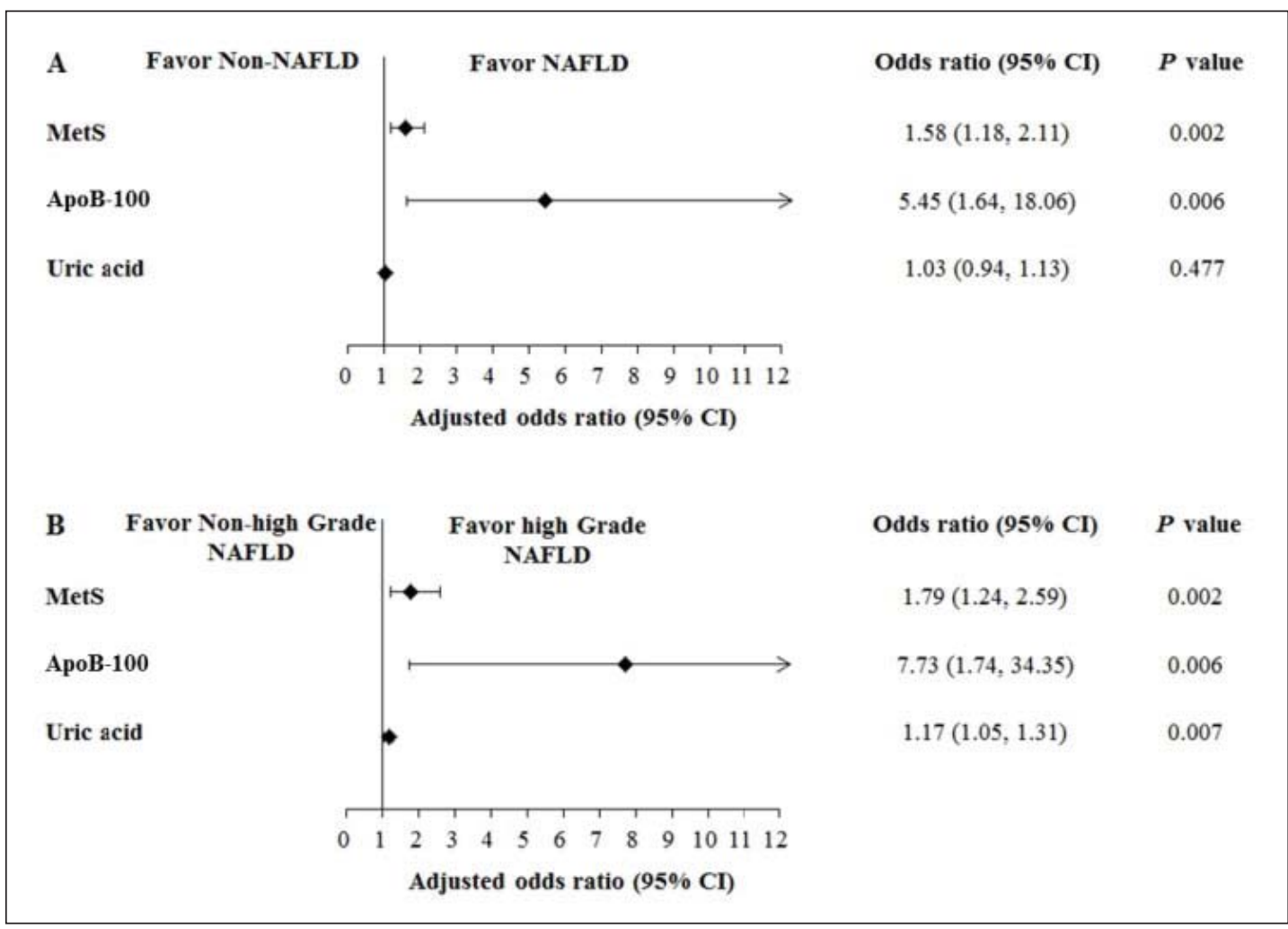

Fig. 4. A Multivariate logistic regression of factors associated with NAFLD. B Multivariate logistic regression of factors associated with a high-grade NAFLD. Adjusted for age, gender, WHR, alanine aminotransferase, aspartate aminotransferase, BMI, metabolic syndrome, gamma-glutamyl transferase, HBV, LDL-cholesterol, total cholesterol; uric acid, creatinine, betel-nut chewing, smoking, ApoB-100.

The prevalence of steatosis in patients with hepatitis C (35.8\% in all patients) was lower than in another study [6]. A relatively lower incidence of steatosis was thought to be related to limited echogenic screening and non-genotype 3 distributions, according to an HCV line probe assay showing that genotypes 1 and 2 were predominant in Taiwan [24].

\section{Factors Associated with NAFLD in HCV}

The prevalence of steatosis in patients with genotype $3 \mathrm{HCV}$ is significantly higher than in patients with other forms of chronic liver disease, e.g., hepatitis B or autoimmune hepatitis, which suggests a direct effect of HCV replication on excess fat accumulation in the liver. The severity of steatosis is associated with the viral load in patients infected with genotype $3 \mathrm{HCV}$, which subsides with sustained response to antiviral therapy but recurs after a virological relapse. In patients infected with other HCV genotypes, a significant relationship exists between steatosis, elevated BMI and waist circumference, which imply the role of host metabolic factors such as insulin resistance. Our study showed that patients with NAFLD who had similar waist circumference, BW, and BMI also had higher fasting blood glucose, TG, and blood pressure as symptoms of metabolic syndrome $(\mathrm{p}=0.001)$. The metabovascular features were still significant in the analyses of various grades of NAFLD. Moreover, the nonsignificant anthropometric measures may be related to the complex mechanisms, including the effect of HCV activity, that contribute to hepatosteatosis. Therefore, underlying dyslipidemia and metabolic disturbance were more essential in the study population; 
ApoB-100 might be a more important predictor of NAFLD in people with a similar waist circumference or BW.

NAFLD in chronic HCV infection increases cardiovascular events and extrahepatic mortality through metabolic disturbances and systemic atherosclerosis, which is induced by excessive oxidative stress and advanced necroinflammation. In patients with CHC infection, hypoadiponectinemia increases hepatic steatosis, inflammation, fibrosis, and hepatocarcinogenesis in animal models and human studies [25]. Interestingly, in our study, hyperuricemia was significantly increased in patients with HCV and advanced steatosis (OR 1.172; 95\% CI 1.047-1.311; $p=0.006$ ), which supported the notion that NAFLD and hyperuricemia are risk markers of hepatic damage, systemic inflammation, and cardiovascular mortality [26]. Taken together, these findings suggest that, in patients with CHC, high-grade NAFLD reflects additional atherogenic risks instead of metabolic syndrome.

\section{Apolipoprotein B-100 on NAFLD}

Apolipoproteins, such as ApoE, ApoB, ApoA1, and several ApoC proteins, are associated with the impact of HCV lipo-viro-particles on viral entry, replication, and infection. The ApoB-100 level was relatively lower in genotype-3 HCV patients, and hypocholesteremia was correlated with the virus load [20].

However, in people with non-genotype $3 \mathrm{HCV}$, hepatosteatosis is primarily related to the host factors of obesity, insulin resistance and DM, and antiviral therapy does not ameliorate steatosis in these patients [26]. Established studies [28-30] showed higher ApoB levels or ApoB/AI ratios associated with fatty liver in patients with DM and in and the general population. Greater ApoB expression is associated with insulin resistance [31], and delayed ApoB clearance is associated with triglyceride lipolysis, greater oxidative stress, and greater glycosylation oxidization of lipoprotein particles [32]. In our study, patients with HCV and NAFLD had typical features of metabolic syndrome, and ApoB-100 was strongly associated with NAFLD after traditional metabovascular factors had been adjusted for; the value of ApoB-100 might be more closely associated with advanced hepatosteatosis (OR 7.73; 95\% CI 1.6434.35; $\mathrm{p}=0.006$ ) in patients with non-genotype $3 \mathrm{HCV}$.

\section{Limitations}

This study has some limitations. First, because of its cross-sectional design, the present findings are inherently limited in their ability to eliminate causal relationships between ApoB-100 and NAFLD. Second, confirmatory HCV testing, e.g., HCV RNA and HCV core antigen testing was not done; thus, we were unable to accurately characterize each patient's carrier status. However, these tests are expensive and infeasible for community health examinations. Third, although a liver biopsy is more sensitive than ultrasonography and is, therefore, the gold standard for this kind of investigation, it is expensive and bears the risks of bleeding and death. Using liver biopsies in population-based studies is unsuitable. Ultrasonography is feasible in a population-based epidemiological study, and interobserver variation can be reduced by experienced operators. Fourth, ApoB- 100 was measured only once in the annual examination, and the coefficients of variability are unavailable because the check-up was unrepeatable. Moreover, we did not test for ApoAI, ApoE, or ApoC because the tests are too expensive and give information similar to that provided by tests of ApoB-100 and the ApoB/ ApoAI ratio [17]. Finally, some medications or diseases related to different lipid profiles and status of anti-viral therapy were missing despite our careful personal medical survey. Additional large prospective investigations of the mechanism are needed to confirm the correlation. 
Lin et al.: Impact of Apolipoprotein B on Hepatosteatosis in a Population Infected with Hepatitis C Virus: A Cross-Sectional Observational Study

\section{Conclusion}

We found a strong association between ApoB-100 and fatty liver in patients with nongenotype $3 \mathrm{HCV}$. Despite metabolic syndrome contributing to hepatosteatosis in the study, ApoB-100 is another independent factor for NAFLD. Physicians should pay more attention to patients with HCV and a high ApoB-100 level; they might indicate advanced steatosis, resistance to antiviral therapy, and poor extrahepatic outcomes.

\section{Disclosure Statement}

No conflicts of interest to declare.

\section{References}

1 Mohd Hanafiah K, Groeger J, Flaxman AD, Wiersma ST: Global epidemiology of hepatitis C virus infection: new estimates of age-specific antibody to HCV seroprevalence. Hepatology 2013;57:1333-1342.

-2 White DL, Ratziu V, El-Serag HB: Hepatitis C infection and risk of diabetes: a systematic review and metaanalysis. J Hepatol 2008;49:831-844.

-3 Machado MV, Oliveira AG, Cortez-Pinto H: Hepatic steatosis in hepatitis B virus infected patients: metaanalysis of risk factors and comparison with hepatitis C infected patients. J Gastroenterol Hepatol 2011;26: 1361-1367.

-4 Butt AA, Xiaoqiang W, Budoff M, Leaf D, Kuller LH, Justice AC: Hepatitis C virus infection and the risk of coronary disease. Clin Infect Dis 2009;49:225-232.

-5 Farrell GC, Larter CZ: Nonalcoholic fatty liver disease: from steatosis to cirrhosis. Hepatology 2006;43(2 suppl 1):S99-S112.

6 Lonardo A, Adinolfi LE, Loria P, Carulli N, Ruggiero G, Day CP: Steatosis and hepatitis C virus: mechanisms and significance for hepatic and extrahepatic disease. Gastroenterology 2004;126:586-597.

7 Sanyal AJ, Contos MJ, Sterling RK, Luketic VA, Shiffman ML, Stravitz RT, Mills AS: Nonalcoholic fatty liver disease in patients with hepatitis $\mathrm{C}$ is associated with features of the metabolic syndrome. Am J Gastroenterol 2003;98:2064-2071.

8 Yoon EJ, Hu KQ: Hepatitis C virus (HCV) infection and hepatic steatosis. Int J Med Sci 2006;3:53-56.

-9 Szanto P, Grigorescu M, Dumitru I, Serban A: Steatosis in hepatitis C virus infection. Response to anti-viral therapy. J Gastrointestin Liver Dis 2006;15:117-124.

10 Targher G, Bertolini L, Padovani R, Rodella S, Arcaro G, Day C: Differences and similarities in early atherosclerosis between patients with non-alcoholic steatohepatitis and chronic hepatitis B and C. J Hepatol 2007;46: 1126-1132.

11 Leandro G, Mangia A, Hui J, Fabris P, Rubbia-Brandt L, Colloredo G, Adinolfi LE, Asselah T, Jonsson JR, Smedile A, et al: Relationship between steatosis, inflammation, and fibrosis in chronic hepatitis C: a meta-analysis of individual patient data. Gastroenterology 2006;130:1636-1642.

-12 Fon Tacer K, Rozman D: Non-alcoholic fatty liver disease: focus on lipoprotein and lipid deregulation. J Lipids 2011;2011:783976.

13 Williams K, Sniderman AD, Sattar N, D’Agostino R Jr, Wagenknecht LE, Haffner SM: Comparison of the associations of apolipoprotein B and low-density lipoprotein cholesterol with other cardiovascular risk factors in the Insulin Resistance Atherosclerosis Study (IRAS). Circulation 2003;108:2312-2316.

14 Onat A, Can G, Hergenç G, Yazici M, Karabulut A, Albayrak S: Serum apolipoprotein B predicts dyslipidemia, metabolic syndrome and, in women, hypertension and diabetes, independent of markers of central obesity and inflammation. Int J Obes (Lond) 2007;31:1119-1125.

15 Relimpio F, Losada F, Pumar A, Mangas MA, Morales F, Astorga R: Relationships of apolipoprotein B(100) with the metabolic syndrome in type 2 diabetes mellitus. Diabet Res Clin Pract 2002;57:199-207.

-16 Walldius G, Jungner I, Holme I, Aastveit AH, Kolar W, Steiner E: High apolipoprotein B, low apolipoprotein A-I, and improvement in the prediction of fatal myocardial infarction (AMORIS study): a prospective study. Lancet 2001;358:2026-2033.

17 Choe YG, Jin W, Cho YK, Chung WG, Kim HJ, Jeon WK, Kim BI: Apolipoprotein B/AI ratio is independently associated with non-alcoholic fatty liver disease in nondiabetic subjects. J Gastroenterol Hepatol 2013;28:678683.

18 Boumaiza I, Omezzine A, Rejeb J, Rebhi L, Kalboussi N, Ben Rejeb N, Nabli N, Ben Abdelaziz A, Boughazala E: Apolipoprotein B and non-high-density lipoprotein cholesterol are better risk markers for coronary artery disease than low-density lipoprotein cholesterol in hypertriglyceridemic metabolic syndrome patients. Metab Syndr Relat Disord 2010;8:515-522. 
Lin et al.: Impact of Apolipoprotein B on Hepatosteatosis in a Population Infected with Hepatitis C Virus: A Cross-Sectional Observational Study

19 Thompson A, Danesh J: Associations between apolipoprotein B, apolipoprotein AI, the apolipoprotein B/AI ratio and coronary heart disease: a literature-based meta-analysis of prospective studies. J Intern Med 2006; 259:481-492.

20 Petit JM, Benichou M, Duvillard L, Jooste V, Bour JB, Minello A, Verges B, Brun JM, Gambert P, Hillon P: Hepatitis $C$ virus-associated hypobetalipoproteinemia is correlated with plasma viral load, steatosis, and liver fibrosis. Am J Gastroenterol 2003; 98:1150-1154.

21 Brunzell JD, Davidson M, Furberg CD, Goldberg RB, Howard BV, Stein JH, Witztum JL: Lipoprotein management in patients with cardiometabolic risk: consensus conference report from the American Diabetes Association and the American College of Cardiology Foundation. J Am Coll Cardiol 2008;51:1512-1524.

22 Bae JC, Rhee EJ, Lee WY, Park SE, Park CY, Oh KW, Park SW, Kim SW: Combined effect of nonalcoholic fatty liver disease and impaired fasting glucose on the development of type 2 diabetes: a 4-year retrospective longitudinal study. Diabetes Care 2011;34:727-729.

-23 Grundy SM, Cleeman JI, Daniels SR, Donato KA, Eckel RH, Franklin BA, Gordon DJ, Krauss RM, Savage PJ, Smith SC Jr: Diagnosis and management of the metabolic syndrome an American Heart Association/National Heart, Lung, and Blood Institute Scientific Statement. Circulation 2005;112:2735-2752.

24 Lee CM, Lu SN, Hung CH, Tung WC, Wang JH, Tung HD, Chen CH, Hu TH, Changchien CS, Chen WJ: Hepatitis C virus genotypes in southern Taiwan: prevalence and clinical implications. Trans R Soc Trop Med Hyg 2006; 100:767-74

25 Liu CJ, Chen PJ, Jeng YM, Huang WL, Yang WS, Lai MY, Kao JH, Chen DS: Serum adiponectin correlates with viral characteristics but not histologic features in patients with chronic hepatitis C. J Hepatol 2005;43:235-242.

26 Petta S, Macaluso FS, Cammà C, Marco VD, Cabibi D, Craxì A: Hyperuricaemia: another metabolic feature affecting the severity of chronic hepatitis because of HCV infection. Liver Int 2012;32:1443-1450.

-27 Patton HM, Patel K, Behling C, Bylund D, Blatt LM, Vallée M, Heaton S, Conrad A, Pockros PJ, McHutchison JG: The impact of steatosis on disease progression and early and sustained treatment response in chronic hepatitis C patients. J Hepatol 2004;40:484-490.

28 Higuchi N, Kato M, Tanaka M, Miyazaki M, Takao S, Kohjima M, Kotoh K, Enjoji M, Nakamuta M, Takayanagi R: Effects of insulin resistance and hepatic lipid accumulation on hepatic mRNA expression levels of apoB, MTP and L-FABP in non-alcoholic fatty liver disease. Exp Ther Med 2011;2:1077-1081.

29 Matsumoto K, Fujita N, Nakamura K, Senoo T, Tominaga T, Ueki Y: Apolipoprotein B and insulin resistance are good markers of carotid atherosclerosis in patients with type 2 diabetes mellitus. Diabetes Res Clin Pract 2008;82:93-97.

-30 Sung KC, Hwang ST: Association between insulin resistance and apolipoprotein B in normoglycemic Koreans. Atherosclerosis 2005;18061-169.

-31 Taghibiglou C, Rashid-Kolvear F, Van Iderstine SC, Le-Tien H, Fantus IG, Lewis GF, Adeli K: Hepatic very lowdensity lipoprotein-ApoB overproduction is associated with attenuated hepatic insulin signaling and overexpression of protein-tyrosine phosphatase $1 \mathrm{~B}$ in a fructose-fed hamster model of insulin resistance. J Biol Chem 2002;277:793-803.

32 Steinberg D: The LDL modification hypothesis of atherogenesis: an update. J Lipid Res 2009;50(suppl):S376S381. 